\title{
Wearable Monitoring and Predicting System for Knee Joint Fatigue Based on Curvature and Pressure Sensing
}

\section{Jiawei Xin}

Fujian Medical University Union Hospital

Jialun Chen

Fujian Agriculture and Forestry University

\section{Xuanyu Huang}

Fujian Medical University Union Hospital

\section{Xiaodong Pan}

Fujian Medical University Union Hospital

Tengyue Zou ( $\nabla$ zouty@fafu.edu.cn )

Fujian Agriculture and Forestry University https://orcid.org/0000-0001-5854-4877

\section{Research}

Keywords: knee joint, fatigue, wearable device, exercise, health

Posted Date: August 13th, 2020

DOl: https://doi.org/10.21203/rs.3.rs-56286/v1

License: (9) This work is licensed under a Creative Commons Attribution 4.0 International License. Read Full License 
2 Wearable Monitoring and Predicting System for Knee Joint

\section{Fatigue Based on Curvature and Pressure Sensing}

4 Jiawei Xin $^{1,2,3}$, Jialun Chen ${ }^{4}$, Xuanyu Huang ${ }^{1,2}$, Xiaodong Pan ${ }^{1,2,3}$, and Tengyue Zou ${ }^{4, *}$

$5{ }^{1}$ Department of Neurology, Fujian Institute of Geriatrics, Fujian Medical University Union Hospital,

629 Xinquan Road, Fuzhou 350001, China; xiaoxinskyhost@163.com

$7 \quad{ }^{2}$ Fujian Key Laboratory of Molecular Neurology, Fujian Medical University, 29 Xinquan Road,

8 Fuzhou 350001, China

$9 \quad{ }^{3}$ Key Laboratory of Brain Aging and Neurodegenerative Diseases, Fujian Medical University, 29

10 Xinquan Road, Fuzhou 350001, China

$11{ }^{4}$ College of Mechanical and Electrical Engineering, Fujian Agriculture and Forestry University,

12 Fuzhou 350002, China

13 * Correspondence: zouty@ fafu.edu.cn

\section{Abstract}

Background: Knee injury is always a trouble for people in daily life. It not only threatens the career of an athlete but also affects a normal engineer through morning running. The injury of the knee joint is found to be directly related to the fatigue caused by excessive exercise.

Methods: An economical embedded system with a designed acceleration-weighted curve fitting method was developed to estimate and predict the knee fatigue state. Then the warning message and recommended lasting time were sent to users to avoid excessive exercise. 24 healthy volunteers were involved in the experiments to verify the effectiveness of the system compared to human perception.

Results: Only using human perception to prevent knee joint fatigue had a risk of failure while the designed wearable system could protect knee successfully. It was also found that 
the knee of female was more likely to be injured than the one of male in intense exercises and a high BMI value could influence the risk of knee injuries during sports. However, a short break in sports could significantly extend the healthy time for knee.

Conclusions: Early warning from the specially designed embedded system can successfully help people avoid knee joint fatigue and injuries during exercises, such as running, badminton, table tennis and basketball.

Keywords: knee joint; fatigue; wearable device; exercise; health

\section{Background}

Knee joint injuries often occur during exercise and cause more than four weeks absence from jobs or team-sports competitions. The anterior cruciate ligament (ACL) lesions and meniscal and medial collateral ligament (MCL) sprains are most common knee joint injuries in daily life. The rate of ACL lesions even reaches 2.8 and 3.2 injury per 10,000 h of exposure in basketball and soccer players from university, respectively [1]. Moreover, ACL rupture usually needs surgical reconstruction and a recovery period over six months which may bring a shadow to the career of an athlete or normal work of an engineer [2]. People's daily strenuous physical exercise without restriction may also cause acute or chronic injury to the knee joint, resulting in body and economic losses. Therefore, prevention of that injury in sports is an important and meaningful work in sports protection. The studies have found that knee injury from sports in closely related to knee joint fatigue caused by excessive exercise [3-5]. Thus, if the knee joint fatigue can be estimated and predicted during the sports, early warning can be made to reduce the intensity or duration of the exercise.

Previous researches have focused on the monitoring of knee joint motion [6]. A wearable goniometer and accelerometer sensory prototype with a Kalman filter for data fusion has been made to measure the flexion-extension angle of knee [7]. In evaluation, the prototype is able to acquire reliable data in dynamic knee movements, but it has not provided an economical embedded solution of portable device for daily application and is without the testing dataset on real sports of human. Another motion capture system based on 17 inertial units and 53 opto-reflective markers is proposed to measure 
the knee adduction and joint contact force during daily living activities of elderly people with knee osteoarthritis [8]. This research proves the feasibility of the inertial motion detecting system but lacks the data analysis for actual daily applications. Furthermore, amounts of sensors applied in the system increase the cost and structural complexity of it, and may make it hard to be adopted wildly in daily life. In order to improve the detecting accuracy and portability of the devices, the new structural sensor made by ordinary fabrics and conductive yarns [9] and mobile communication technique [10] are also involved in. The ground reaction forces of ski jump landing related to knee are studied using wearable sensors [11]. In that study, the plantar force insoles are combined with inertial motion units to determine the possible relationship of those forces. However, none of these studies linked the movement and stress of the knee joint to the fatigue judgment of knee, which is the key to knee protection.

In order to keep health of knee in sport competition and daily exercise, sensoring equipment and an estimated system are built in this paper to help people control their exercise intensity and duration. The primary contributions of this report can be described as follows: (1) link the knee injuries to its fatigue and propose a comprehensive load formula for knee joint fatigue estimation; (2) the acceleration of the end of the tibial which reflects the active intensity of the muscles around the knee joint is measured as an additional weight for curve fitting and a proportional-integral controller is also involved to increase the predicting accuracy; and (3) an economical sensoring system based on ST Microelectronics (Geneva, Switzerland) STM32F103 platform is developed to validate the effect of the algorithm and be a prototype for daily use. The remainder of this report is organized as follows: after reviewing related knee joint physiology in Section 2, we provide a fatigue judgement algorithm for knee joint in Section 3. In Section 4, a real-time embedded hardware is built to sense the load of knee joint during exercise. The experimental results are presented in Section 5. Finally, the conclusions are stated in Section 6.

\section{Results}

Figure 1 shows the flexion angle and plantar pressure data during running and playing basketball acquired by the devices and presents the illustration of cubic polynomial fitting estimation for knee 
fatigue. It can be found that the degree of knee fatigue increased with time during the exercises and the designed system is successful to estimate the fatigue of knee joint in real time. Figure 2(a) shows the healthy time of different genders for knee joint during the four exercises without break which can keep the fatigue degree of knee less than 80 present. It is obvious that the knee of female is more likely to be injured during these exercises and the tendency is more significant in sports like basketball and running in which the movements are more intense in the lower limb. Figure 2(b) shows the healthy time of different BMI situation during the exercises without break. When playing badminton and table tennis, the participants with BMI value less than 24 are able to have more time to stay healthy for knee joint. The difference is not significant in playing basketball and running although their mean values have the same tendency. This may be due to the large time variance caused by the deviation of people's personal tolerance to playing basketball and running. Thus, the people with larger BMI value may have higher risk of knee injuries during playing badminton and table tennis. This may be due to the fact that excessive body weight puts more loads on the leg muscles during exercise. As shown in Figure 2(c), the short break during sports can significantly extend the healthy time for these exercises and avoid knee injuries efficiently; conversely, long-term high-intensity continuous exercise will increase the risk of knee injury. After 14 days of first stage testing, none of the participants reported discomfort or pain in their knee joint.

In the second stage of experiment, the testing group equipped the sensoring device for warning fatigue of knee joint while the control group depended on the feeling of themselves. As shown in Figure 3(a) and (b), the Lysholm scores and IKDC scores of control group were significantly decreased after running and playing basketball while the ones of testing group did not changed obviously. This proved that only using human perception to prevent fatigue of knee joint and avoid injury had a risk of failure; and the use of a suitable electronic predicting system could achieve this goal. Because playing basketball is more intense than running for knee joint, the reduction in scale scores is more significant, and the changes in Figure 3(a) and (b) conformed to this trend. Figure 3(c) and (d) shows the Lysholm sores and IKDC scores of control group for different genders before and after playing basketball and running. It can be found that female suffered more decrement in scale 

sores than male during these two sports, which implied that women is more dangerous to be injured in 106 knee than man in these sports. This trend is consistent with the conclusion in the first stage of 107 experiment. Figure 3 (e) and (f) also shows that the BMI value could influence the risk of knee injuries 108 during sports.

(a)

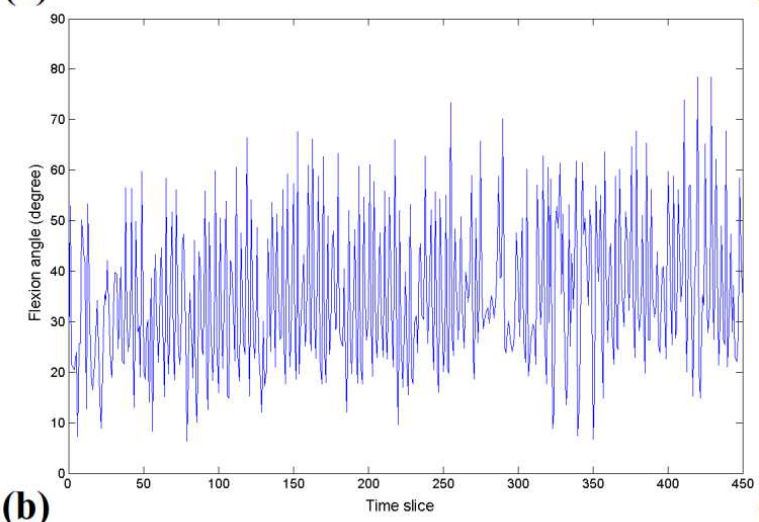

(b)

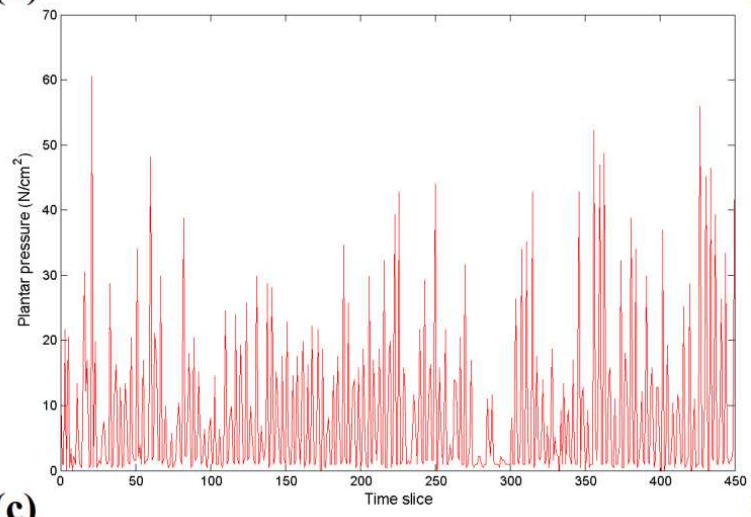

(c)

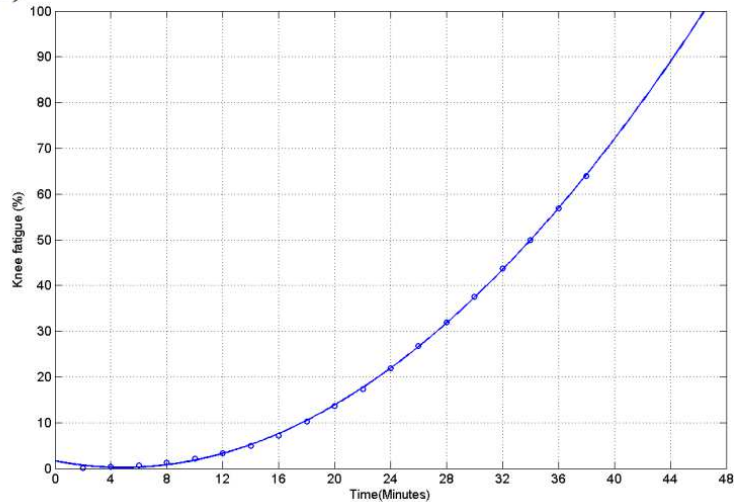

(d)

(e)
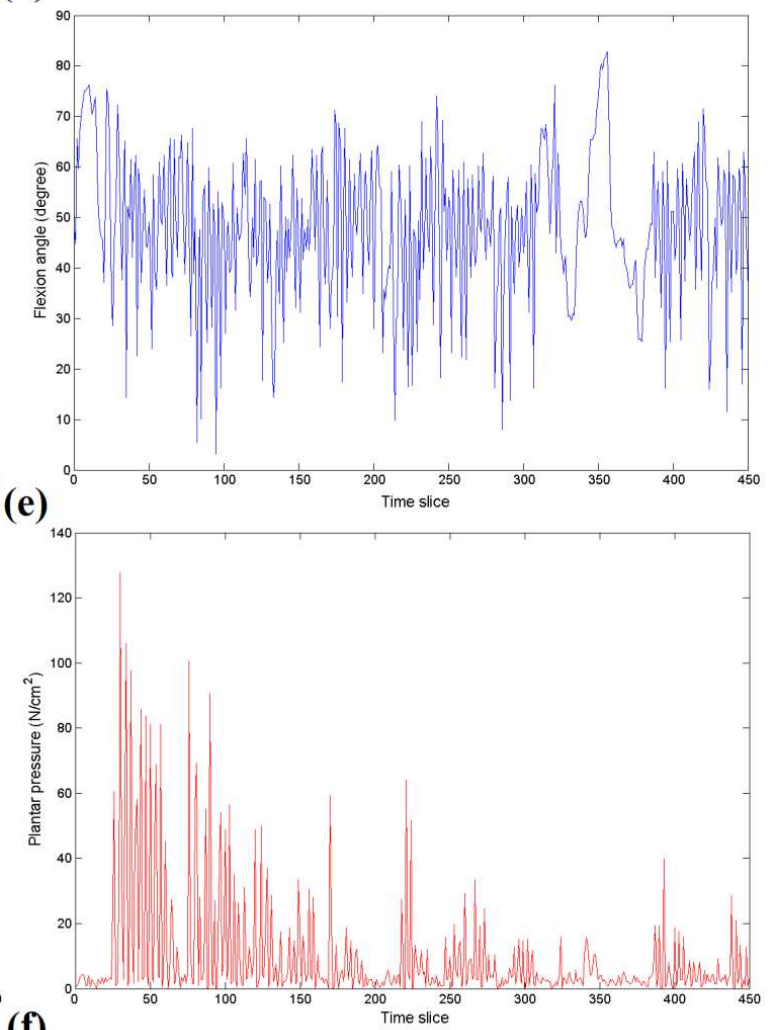

(f)

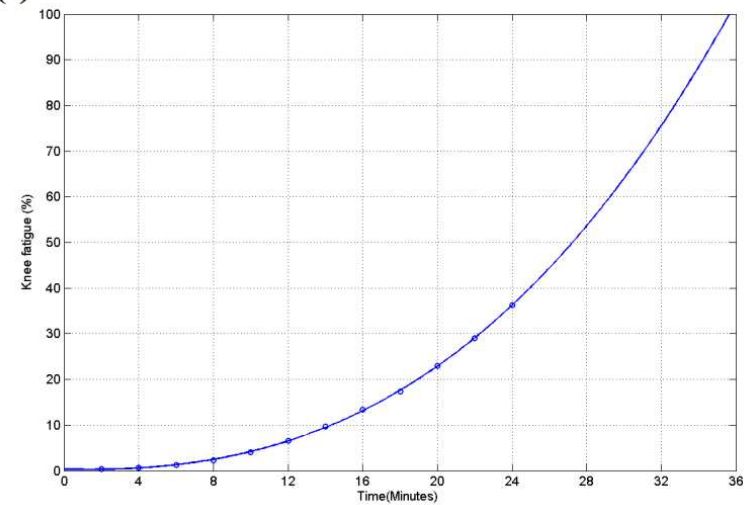

110 Figure 1. (a) Illustration of flexion angle data acquired by the device in running; (b)

111 Illustration of plantar pressure data acquired by the device in running; (c) Illustration of 112 predicting curve of knee fatigue in the 38th minute for running; (d) Illustration of flexion 113 angle data acquired by the device in playing basketball; (e) Illustration of plantar pressure data 
(a)

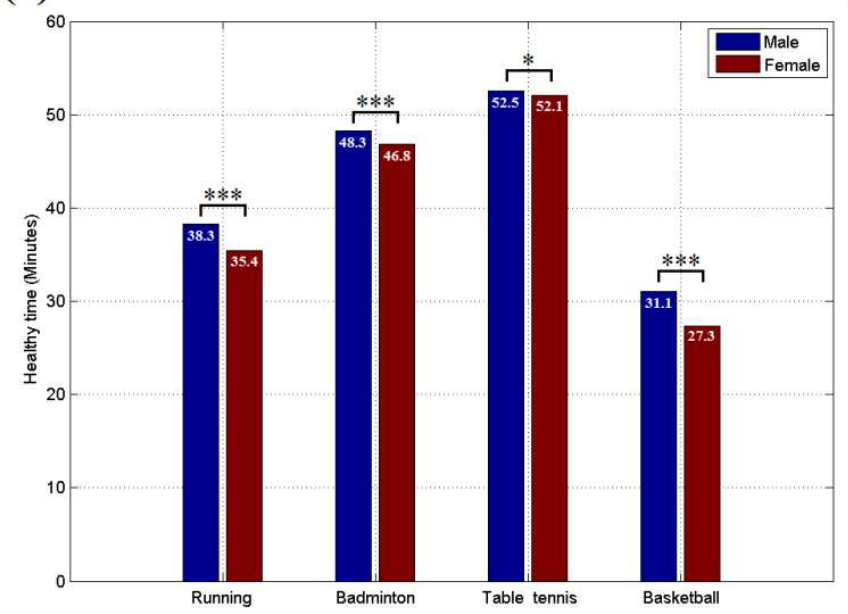

(b)

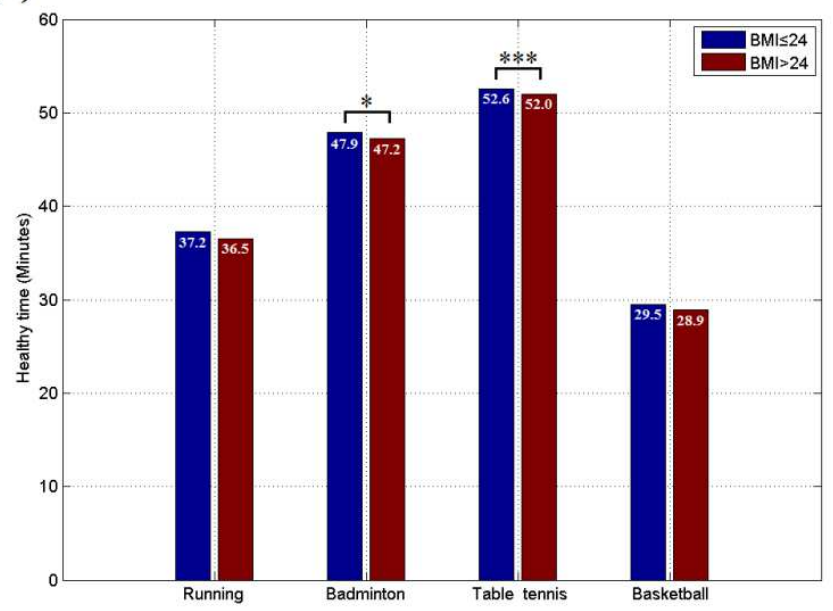

(c)

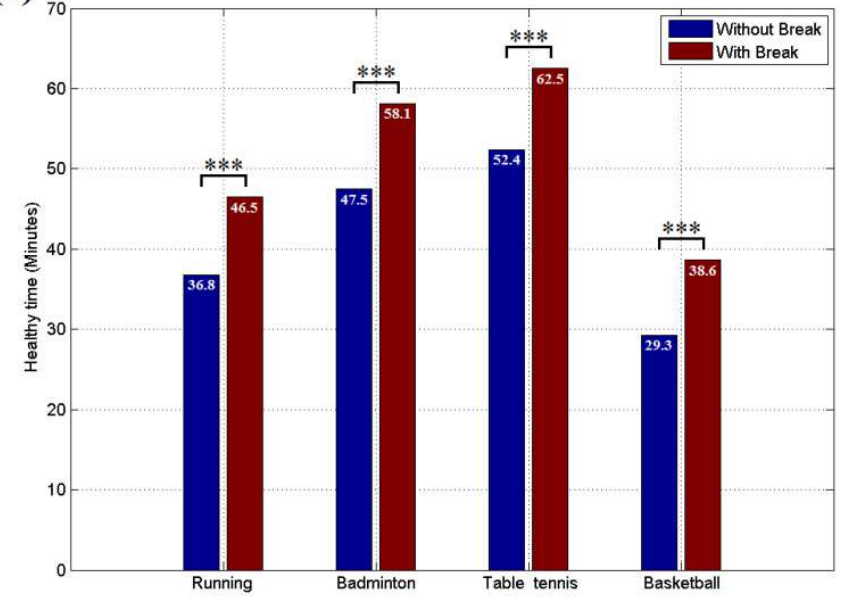

117 Figure 2. (a) The healthy time of different genders for knee joint during the four exercises

118 without break; (b) The healthy time of different BMI situation for knee joint during the four

119 exercises without break; (c) The healthy time for knee joint during the four exercises with and without break. $(* p<0.05, * * p<0.01, * * * p<0.001)$ 
(a)

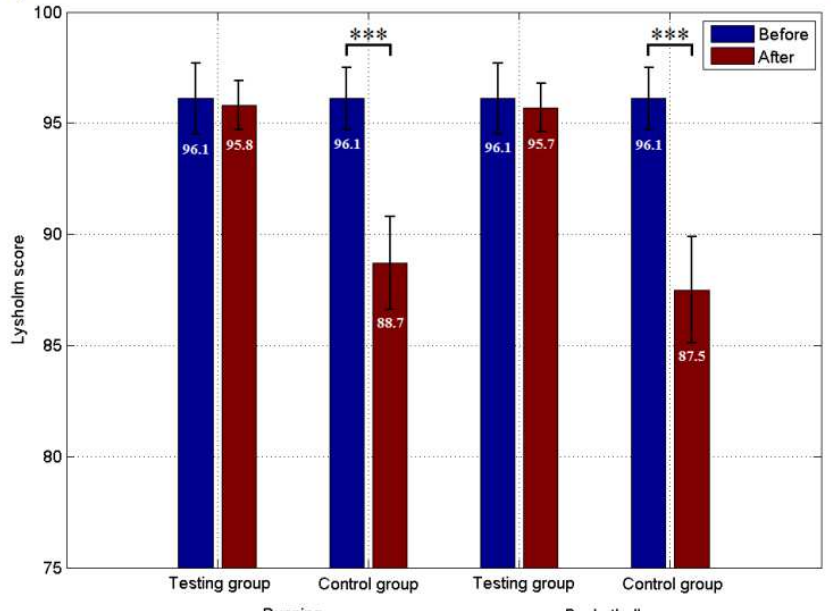

(c)

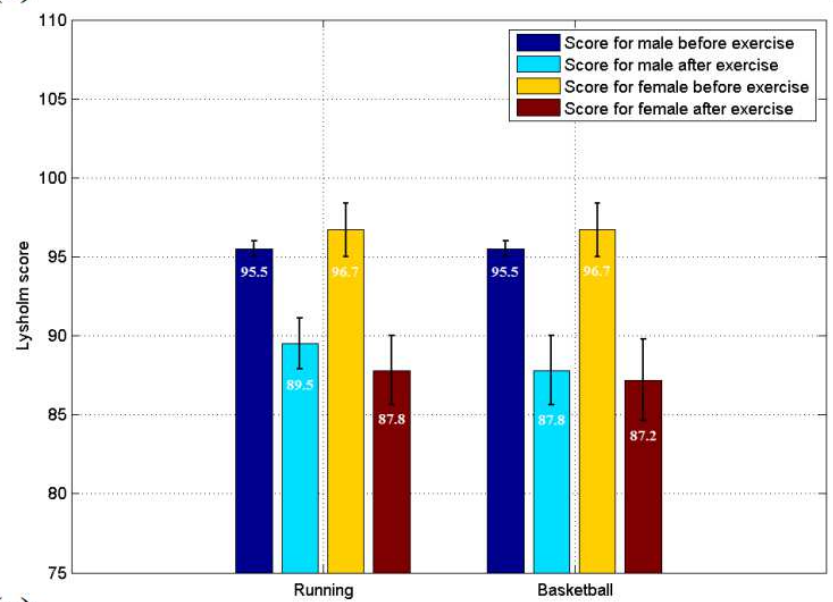

(e)

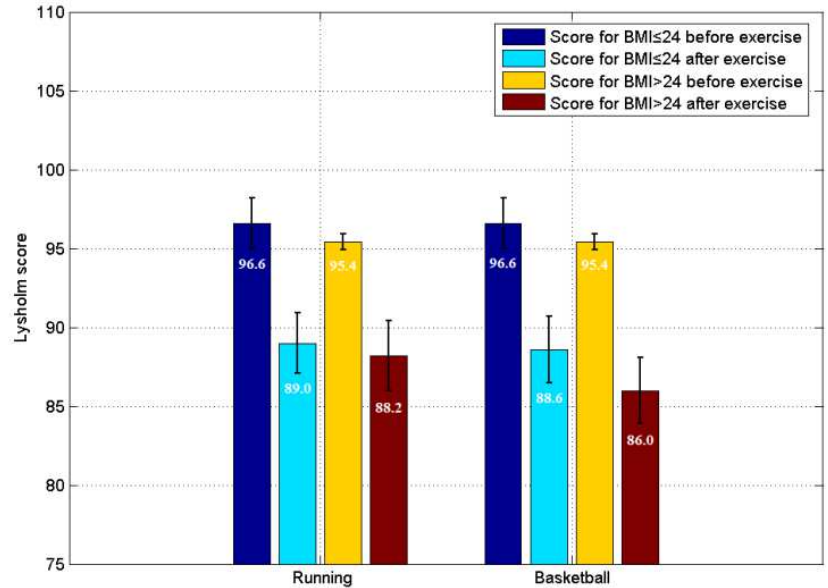

(b)

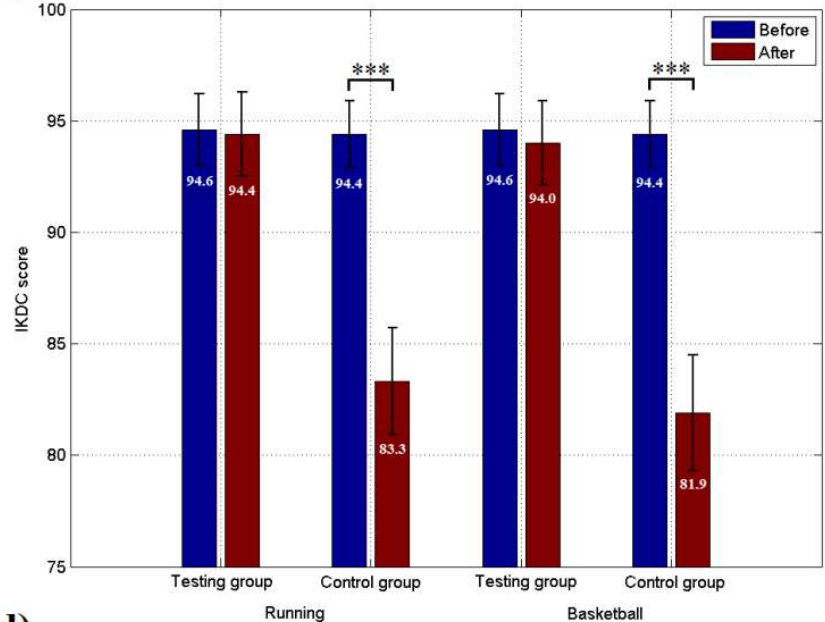

(d)

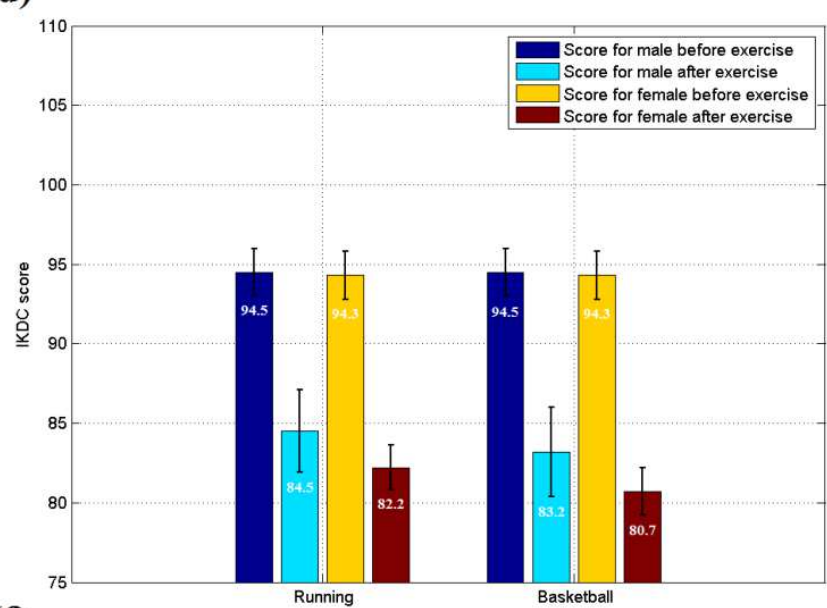

(f)

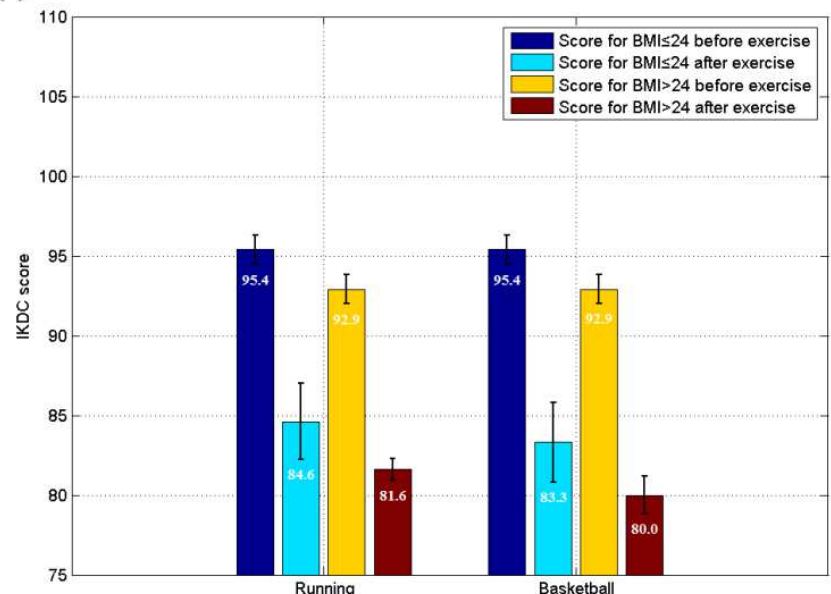

Figure 3. (a) Lysholm sores of testing and control groups before and after playing basketball and running; (b) IKDC sores of testing and control groups before and after playing basketball and running; (c) Lysholm sores of control group for different genders before and after playing basketball and running; (d) IKDC sores of control group for different genders before and after playing basketball and running; (e) Lysholm sores of control group for different BMI situation before and after playing basketball and running; (f) IKDC sores of control group for different 
BMI situation before and after playing basketball and running. $(* p<0.05, * * p<0.01, * * * p$ $<0.001)$

\section{Discussion}

According to the experimental results, the designed estimation and predicting algorithm has ability to warn the risk of knee joint injuries for users during exercises. The economical electronic prototype 133 of sensoring system is able to acquire the data successfully in real time which can help to make a 134 correct decision. It can also be found in Figure 2 and 3 that playing basket had the greatest risk of knee 135 fatigue in these four sports due to its fast running and strong physical confrontation while playing table 136 tennis had the lowest risk, which is consistent with previous report [3]. Furthermore, female gender 137 and high BMI value are two factors to increase the risk of knee injuries. From the scale scores of 138 participants, the designed sensoring system is proved to be able to prevent exercisers from knee joint 139 injuries caused by fatigue. However, only using human perception to prevent fatigue of knee joint and 140 avoid injury during exercise has a risk of failure.

\section{4. Conclusions}

In order to reduce the risk of knee injuries during exercise, a wearable system with STM32 microcontroller and multiple sensors was developed to acquire the parameters of knee joint. Then an 144 acceleration-weighed curve fitting estimation was proposed to estimate the fatigue state. According to 145 the experimental results, the system is helpful to prevent knee joint from injuries induced by excessive 146 exercise and lead the users to take a suitable exercise rhythm, intensity and time.

\section{Materials and Methods}

148 Because the fatigue state of knee joint is affected by the exercise load and the degree of flexion and 149 extension, it can be estimated and predicted according to the moving parameters. In this paper, 150 exercise load and flexion angle of knee joint are taken into consideration, and an acceleration-weighted 
151 curve fitting method is introduced to estimate and predict the percentage of knee fatigue. Then the 152 excessive load during exercise is able to be avoided and the knee joint can be protected.

\subsection{Mechanism and Characterization of Knee Injury}

The knee joint is composed of the lower end of the femur, the upper end of the tibia and the patella with many ligaments and muscles around it. The main ligaments are medial collateral ligament (MCL), lateral collateral ligament (LCL), and anterior cruciate ligament (ACL), posterior cruciate ligament (PCL), etc. Although the fibula does not participate in the formation of the knee joint, it has a role in the stability of the knee joint that cannot be ignored because of the origin of the lateral collateral 159 ligament [12]. Stability maintaining mechanism of knee joint includes active stabilizing structure, passive stabilizing structure and innervation [13]. The active stabilization structure includes the muscles and skin around the knee joint; the passive stabilization structure includes the knee ligament, joint capsule, meniscus, articular cartilage, etc. Both of them are under the control of the nervous system to jointly maintain the stability of the knee joint. Passive stabilization structure is the basis for maintaining knee joint stability, so that the structure of each part of the knee joint moves according to a certain direction with participation of the active stabilization structure and innervation.

The knee joint is an approximate ginglymus, whose range of flexion and extension in the sagittal 167 plane is larger, while the range of motion in the other two planes is smaller [14]. The range of motion 168 of a normal knee joint is defined as the steady range of knee, that is, the range of motion of normal 169 adduction, abduction, internal rotation, external rotation, and hyperextension. When the range of 170 motion exceeds the normal one, the knee joint at this time is considered to be in an unstable state 171 called instability. Injuries to the knee include bone, cartilage, and ligament injuries. There are many 172 ligament injuries, and bone or cartilage injuries are generally combined with one or several ligament 173 injuries $[1,15]$.

174 Common knee injuries include ligament injury, meniscus injury, and patella strain [13]. Ligament 175 injuries mostly occur in basketball, football and other ball sports, as well as gymnastics and running 176 [16]. In these exercises, the knee joint is often in a state of flexion. The calf suddenly abducts and 
externally rotates, or the foot and calf are fixed, and the thigh suddenly rotates and adducts. This is very likely to cause damage to the medial collateral ligament of the knee joint. Most of the collateral ligaments are damaged at the same time as the joint capsule, cruciate ligament, or meniscus. After the injury, the knee joint was partially swollen, and the flexion and extension function was limited. The meniscus injury is due to the inconsistent movement of the medial and lateral meniscus when the knee is suddenly extended from the flexed position. The misalignment of the thigh and calf positions will squeeze the meniscus, causing tearing or abrasive chronic injury of the meniscus. Furthermore, the patella strain is mainly due to the repeated flexion and extension of the knee joint, which causes the corresponding joint surface of the patella and femur to be abnormally misaligned, and the impact of the twisting and friction causes local tissue and cell metabolism abnormalities.

Excessive exercise and fatigue of the knee joint have been shown to significantly increase the risk of knee injury $[4,17]$. While the knee joint is gradually fatigued, the flexion angle and frequency of its movement will slightly decrease [1]. By observing the changes of these external parameters, combined with the corresponding exercise data, the progressive degree of knee fatigue can be analysed and predicted, then the exercise plan is able to be adjusted in time to reduce the risk of knee injuries.

\subsection{Comprehensive load for knee joint}

The knee joint fatigue is closely related to the contact force, flexion angle and moving frequency of the joint. During the exercise, the greater the contact force, the larger the flexion angle, and the higher the moving frequency is, the faster the knee joint will be fatigue. Thus, a comprehensive load formula is proposed to estimate the total burden of knee joint during sports involving the three factors as show in Equation (1).

$$
l^{t}=\varepsilon \cdot k^{\rho \cdot \Delta t_{n r} / \Delta t_{g}} \cdot b^{(B M I-\delta)} \cdot\left(P-P_{b m}\right) \cdot\left(C-C_{b m}\right)
$$

where $l^{t}$ is comprehensive load at time slice $t ; P$ represents the maximum plantar pressure acquired by sensors on the insoles in a small sampling time window around the time slice $t$, which reflects the contact force of knee joint; $P_{b m}$ denotes the benchmark value of plantar pressure which is sampled during standing still of user; $C$ is the maximum radian of flexion angle obtained by flex sensors in a 
small sampling time window around the time slice $t ; C_{b m}$ is the benchmark value of flexion angle 203 sampled during standing still of user; BMI represents body mass index, which is currently the most 204 commonly used tool in the world to measure whether a person is too thin or too fat. The highest BMI 205 value for normal weight is usually 25 in the world and 24 in China; $\delta$ is an adjustment parameter specified by the user; $k$ denotes the base of an exponential function used to adjust the shape of the curve; $\Delta t_{n r}$ represents the interval time of knee joint flexion sampled under usual walking state of the user; $\Delta t_{g}$ is the interval time between the last knee flexion and the current one, used to describe the 209 frequency of knee motion; $\rho$ is an adjustment factor, specified by the user; $b$ denotes the base of 210 another exponential function used to adjust the shape of the curve; $\varepsilon$ is also an adjustment factors,

211 specified by the user. Before each experimental test, there needs to be a calibration procedure to 212 acquire the parameter $\Delta t_{n r}, P_{b m}$ and $C_{b m}$ automatically from the user for the system through standing 213 still and normal walking.

$$
s^{t}=\int_{0}^{t} l^{t} d t
$$

214 As shown in Equation (2), the comprehensive load $l^{t}$ accumulates over time to make total load $s^{t}$ 215 and the increase of flexion angle, plantar pressure and motion frequency will accelerate accumulation 216 of the value.

\section{5.3. Acceleration-weighted curve fitting estimation}

218 Because the knee fatigue is closely related to the active intensity of the muscles around the joint, the 219 acceleration of the end of the tibial, which is measured by the acceleration sensor MPU-6050, can be 220 viewed as an indicator of the muscle activity in the exercises. A higher acceleration value may increase 221 the fatigue of the knee joint. Thus, this value can be taken as a weight in the fitting estimation. A 222 proportional-integral (PI) controller [18] is introduced to involve the influence of strenuous muscle. In 223 addition to affecting curve fitting through proportional term, continuous intense muscle movements 224 also produce cumulative effects through integral term. The longer the duration, the greater the 225 cumulative impact on the knee fatigue. As shown in Equation (3), $\Delta a(t)$ represents the acceleration 
226 redundancy at time $t$ which exceeds the threshold $a_{t h r}$, and it can be got in calibration procedure under

227 normal walking of user . Equation (4) shows the computational formula of PI controller.

$$
\begin{gathered}
\Delta a(t)=a(t)-a_{t h r} \\
w(t)=K_{P}\left[\Delta a(t)+\frac{1}{T_{I}} \int_{0}^{t} \Delta a(\tau) d \tau\right]
\end{gathered}
$$

228 where $K_{P}$ represents the proportional gain, $T_{I}$ denotes the integral time and $w(t)$ is the acceleration 229 redundancy at time $t$ which can be used as weight in the curve fitting. Because the Equation (4) can 230 only be used in analogue systems, the integral component should be discretized for the digital 231 equipment. Equation (5) shows the formula for conversion from the integral term to the sum of discrete 232 errors:

$$
\int_{0}^{t} \Delta a(\tau) d \tau=\sum_{j=0}^{n} \Delta a(j) \cdot \Delta t=T \sum_{j=0}^{n} \Delta a(j)
$$

233 where $\Delta t=T$ represents the sampling period. In our experiments, the value of $T$ is set to 1 . Equation (5) 234 can then be rewritten in discrete form as Equation (6):

$$
w(k)=K_{P}\left[\Delta a(k)+\frac{T}{T_{I}} \sum_{j=0}^{n} \Delta a(j)\right]
$$

According to Equation (7), Equation (6) can be further converted to the incremental form as 236 Equation (8). That formula can simplify the calculation and save storage space.

$$
\begin{aligned}
& w(k)-w(k-1)=K_{P}\left[\Delta a(k)-\Delta a(k-1)+\frac{T}{T_{I}} \Delta a(k)\right] \\
& w(k)=w(k-1)+K_{P}[\Delta a(k)-\Delta a(k-1)]+K_{I} \Delta a(k)
\end{aligned}
$$

237 where $K_{I}=K_{P}\left(T / T_{I}\right)$ is the integral coefficient. Then each $w(k)$ should be standardized within the array $238 W$ which is consist of all the $w(k)$ values following Equation (9), where $\operatorname{Max}(W)$ represents the 239 maximum element inside the array and $\operatorname{Min}(W)$ is the minimum one. Furthermore, the array $W_{\text {norm }}$ is 240 made of all the $w_{\text {norm }}(k)$ values and can be used for the next weighted curve fitting step.

$$
w_{\text {norm }}(k)=\frac{w(k)}{\operatorname{Max}(W)-\operatorname{Min}(W)}
$$


After obtaining the normalized acceleration redundancy $w_{\text {norm }}(\mathrm{i})$, the weighted curve fitting is able 242 to be run on the database to figure out the prediction of knee fatigue using Equation (10).

$$
\begin{gathered}
\hat{w}=\left(S^{T} W_{\text {norm }} S\right)^{-1} S^{T} W_{\text {norm }} F \\
W_{\text {norm }}=\left[\begin{array}{cccc}
w_{\text {norm }}(1) & 0 & \ldots & 0 \\
0 & w_{\text {norm }}(2) & & \\
\vdots & & \ddots & \\
0 & & & w_{\text {norm }}(i)
\end{array}\right]
\end{gathered}
$$

243 where $\hat{w}$ represents the fitting coefficient; $S$ denotes the vector of total load $\mathrm{s}^{i}$ at each point; $F$ is the

244 vectors of knee fatigue percentage $f^{i} ; W_{\text {norm }}$ is a weight diagonal matrix as shown in Equation (11) 245 containing acceleration redundancy $w_{\text {norm }}(\mathrm{i})$ as its elements.

\section{5.4. Fatigue judgement and predication}

247 Due to the fact that knee fatigue will be reflected in the peak knee flexion angle [1], the angle can 248 be used to help estimate the degree of knee fatigue. However, a key function of the system is to 249 calculate the exercise load on the knee joint and evaluate the suitable duration for different exercises. 250 Thus, the system needs to find the mapping from the total load $s^{t}$ to the percentage of knee fatigue $f^{t}$ at 251 time slice $t$ using the peak flexion angle as a tool. From the initial moment to the latest moment in 252 which the knee joint is fatigue, the peak knee flexion reduces nearly 10 degree [1]. According to this 253 basis, the mapping from the total load to the percentage of knee fatigue can be built in a calibration 254 procedure. In that procedure, let the participant do sport until he feels tired of his knee joint. During 255 that process, at the exact time of each 1 degree reduction of the peak knee flexion, the corresponding 256 total load $s^{i}$ is calculated, where $i$ is a serial number. Then the percentage of knee fatigue $f^{i}$ is estimated 257 by the peak knee flexion degree and the pair $\left(s^{i}, f^{i}\right)$ can be viewed as a point in the mapping 258 relationship. After acquiring these $\left(s^{i}, f^{i}\right)$ key points, the cubic polynomial fitting can be performed to 259 finish the whole curve of the mapping from $s^{t}$ to $f^{t}$. 


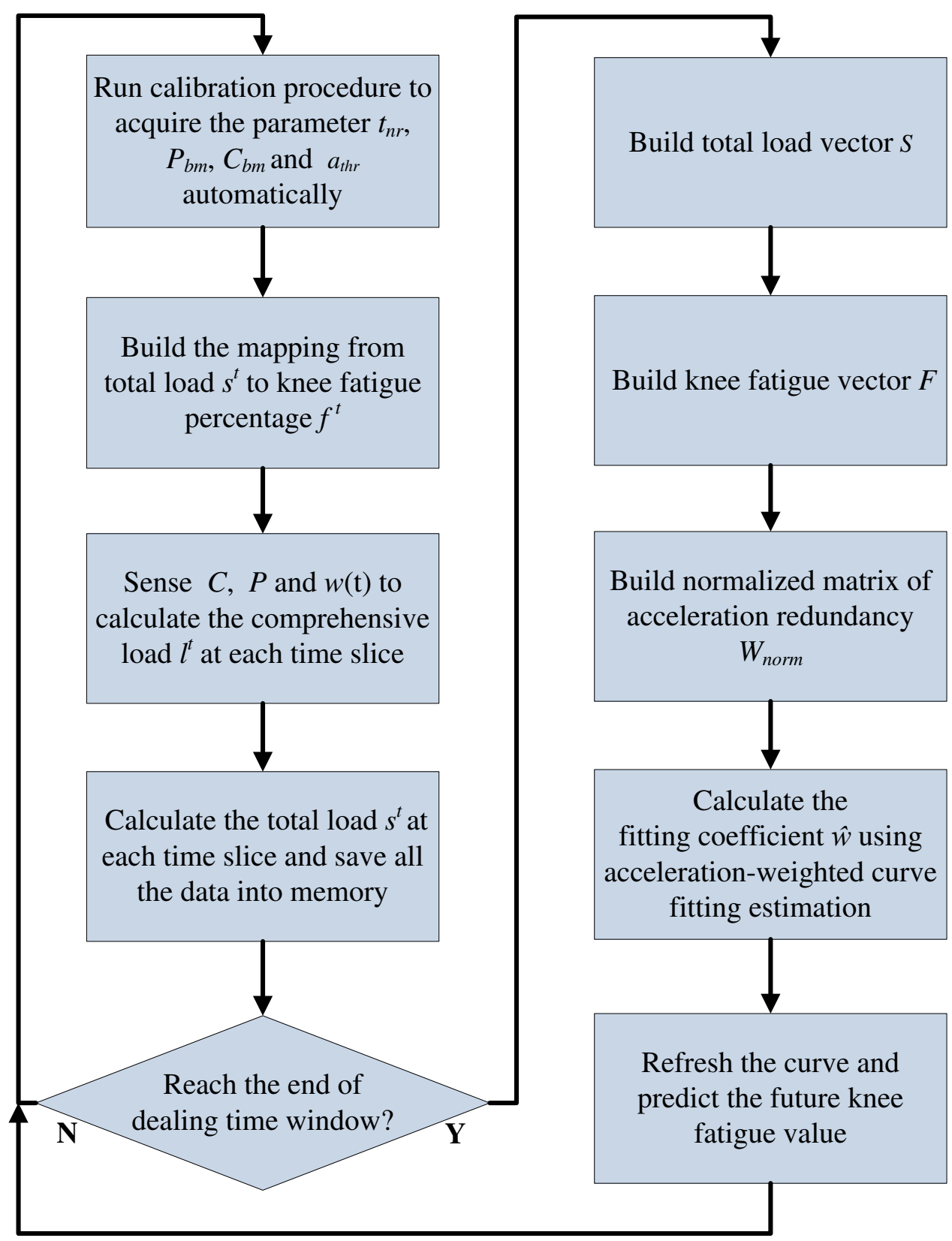

Figure 4. The sensing and predicting procedure for knee fatigue judgment.

The whole sensing and predicting procedure is shown in Figure 4 for getting the fitting coefficient

$263 \hat{w}$. After the calibration procedure and mapping building, $C, P$ and $w(t)$ are sensed by the device to 264 evaluate the current percentage of knee fatigue. For that purpose, a dealing time window, which can be 265 set to $1,2,3$ or 5 minutes in usual, is needed for finding key points in the estimation. At the end of 266 each window, the data acquired before will be calculated to find the fitting coefficient $\hat{w}$ and the 267 predicting curve will be refreshed. Then the healthy remaining time $t_{\text {rem }}$ which indicates the suitable 268 time left for the current exercise state without knee injury can be estimated by Equation (12), where $269 f^{\text {end }}$ represents the final fatigue state of knee decided by user; the $\hat{w}$ is the fitting coefficient; the $s^{\text {now }}$ is 
270 the total load at this moment and $l^{\text {now }}$ denotes the comprehensive load value at current time. The

271 healthy remaining time $t_{r e m}$ can be used to warn the user that how much time is left before knee injury 272 under the current exercise burden.

$$
t_{\text {rem }}=\left(f^{e n d} / \hat{w}-s^{\text {now }}\right) / l^{\text {now }}
$$
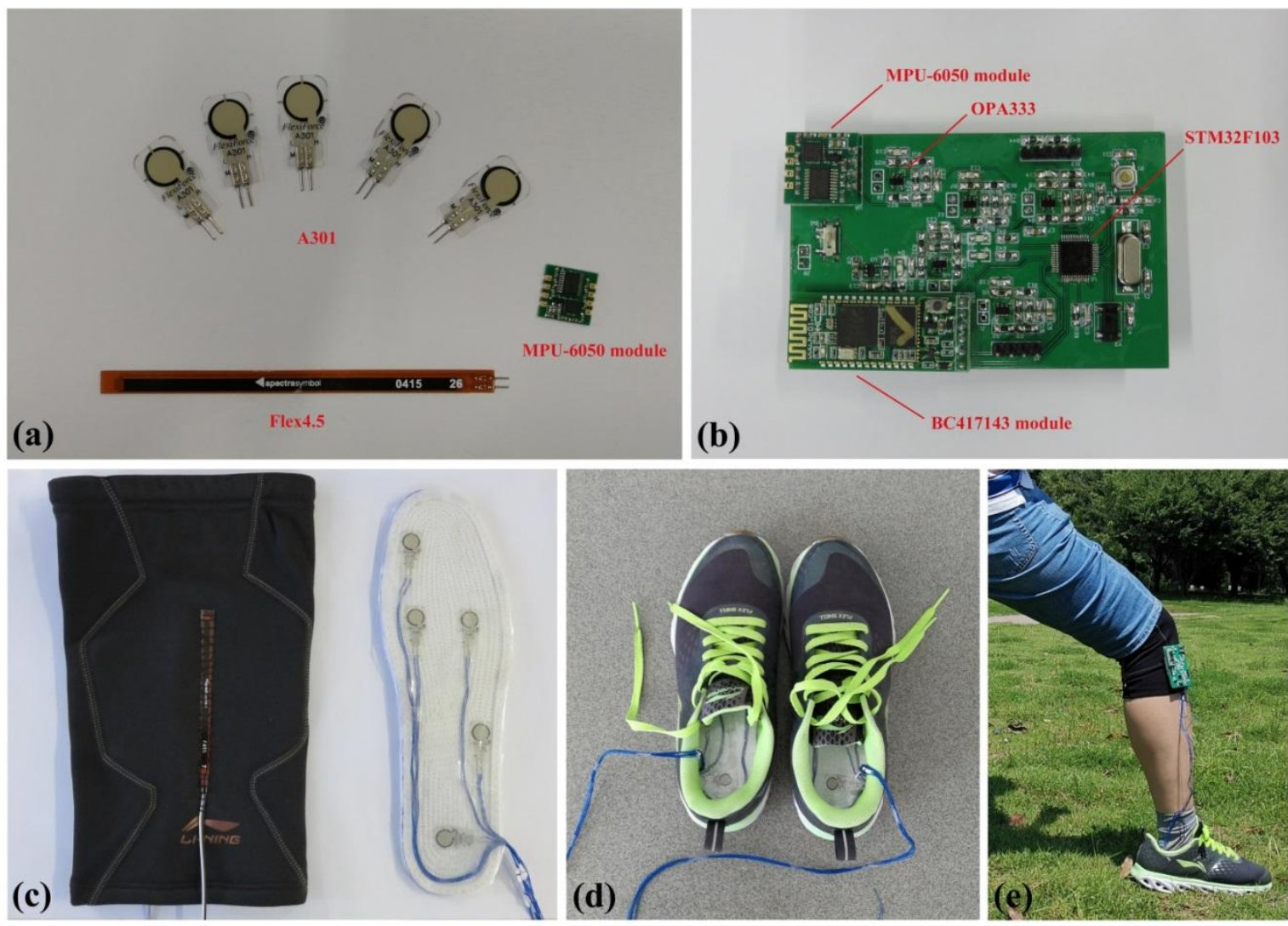

Figure 5. (a) Illustration of the three kinds of sensors used in the sensoring device; (b) Illustration of the hardware of the wearable device; (c) Illustration of the wearable accessories with sensors of the device; (d) Illustration of the shoes with sensors of the device; (e) Illustration of the wearing situation 277 of the sensoring device.

\subsection{Wearable Sensoring Device for Knee Joint}

In order to acquire the exercise data in real time, a wearable sensoring device is implemented on the 280 STM32F103 microcontroller platform from ST Microelectronics Corporation (Geneva, Switzerland). 281 As shown in Figure 5(a) three kinds of sensors are adopted in the device. The Flexiforce A301 is a 282 pressure sensor from Tekscan Corporation (Boston, US) with only $0.203 \mathrm{~mm}$ thickness and less than 5 283 ms response time, which can be deployed on the insole of the shoe to measure the plantar pressure as 
shown in Figure 5(c) and (d). A Flex Sensor 4.5" from Spectra-Symbol Corporation (Salt Lake City,

US) is used to reflect the flexion angle of knee joint. An MPU-6050 module from InvenSense

286 Corporation (Sunnyvale, US) containing a 3-axis gyroscope and a 3-axis accelerometer is adopted to acquire the acceleration of the end of the tibial for weighted curve fitting. The hardware of the sensoring device is shown in Figure 5(b), in which the integrated operational amplifier OPA333 from Texas Instrument (Dallas, US) is designed to regulate the input signal. A BC417143 Bluetooth module from Cambridge Silicon Radio (Cambridge, UK) is used to send messages out through wireless. The 291 Figure 5(e) shows the wearing situation of the device and with the help of it the parameters of knee joint can be measured during sports and the knee fatigue state is able to be estimated.

\subsection{Experiment and participants}

The experiments were performed involving 24 healthy volunteers (12 males, 12 females; for male, age $=22.9 \pm 0.8$ years, height $174.5 \pm 3.3 \mathrm{~cm}$, body mass $67.1 \pm 6.8 \mathrm{~kg}$; for female, age $=23.2 \pm 0.9$ years, height $162.9 \pm 2.9 \mathrm{~cm}$, body mass $55.4 \pm 6.9 \mathrm{~kg}$ ) without heart disease, nervous system disease, unsound limbs, etc. Four popular exercises in daily life are adopted for the tests, including running, badminton, table tennis and basketball. Participants were excluded if they had any pain (acute or chronic) during sports. Flexion angle of knee, plantar pressure and the acceleration of the end of the tibial were sensed by the device and involved in the estimation and predication of knee fatigue after 301 mean filtering. For the experiments, the parameters of the system were set as follow: $\varepsilon=\rho=1, k=b=e$ (the natural logarithm), $\delta=24, K_{P}=3, T_{I}=6, T=1$ for the $w(k)$ calculation, the sampling time window was set to 1 second and the dealing time window was set to 2 minutes. A calibration procedure was performed before testing to get parameter $\Delta t_{n r}, P_{b m}, C_{b m}, a_{t h r}$ and build the mapping from total load to knee fatigue percentage. The designed system showed warning messages to participants when the fatigue degree reached 60 percent, provided the estimated left time for healthy exercise, and the 307 participants stopped the exercise at 80 percent of the knee fatigue. There are two kinds of test mode, 308 the test without break and with break. In the mode without break, the participants do continuous exercise without break until stopping time. While, in the mode with break, the participants make a 5- 
310 minute break after 20 minutes of exercise and continue to do the exercise. The exercising time for

311 these separate parts is added to be counted as the total duration.

312 The whole procedure of experiment was divided into two stages. In the first stage, all the 313 participants were organized into a same group and equipped fatigue predicting devices for warning. All

314 the participants were asked to attend those four kinds of exercises every day in the two test mode with 315 full recovery and the whole testing procedure lasted two weeks. During running exercise the 316 participants were asked to keep a $2.3 \mathrm{~m} / \mathrm{s}$ pace. The predicting capability of the device, the gender317 comparable situation, and the influence of BMI and break during exercises were studied in this stage.

318 In the second stage, the participants were divided into two groups, testing group (6 males, 6 females; 319 age $=23.2 \pm 0.7$ years, height $168.9 \pm 6.7 \mathrm{~cm}$, body mass $61.0 \pm 9.1 \mathrm{~kg})$ and control group $(6$ males, 6 320 females; age $=22.9 \pm 0.9$ years, height $168.5 \pm 6.3 \mathrm{~cm}$, body mass $61.5 \pm 8.8 \mathrm{~kg}$ ). The participants in 321 testing group equipped the sensoring devices and stopped the exercise at 80 percent of the knee fatigue. 322 However, the participants in control group had not stopped their exercise until they felt tired in leg or 323 knee by themselves. All the participants were asked to play basketball at least once every day and that 324 procedure lasted two weeks with the same parameters of the first stage but without break. Data were 325 acquired through the designed hardware and the average values were taken for analysis. Moreover, the 326 Lysholm score [19, 20] and the International Knee Documentation Committee (IKDC) score [21, 22] 327 of the participants were judged by two independent experienced orthopedists from the Department of 328 Orthopedics, Fujian Medical University Union Hospital. After that, the participants were given a three329 month rest to restore their knee joints for the next running test. Before the running test, these two scale 330 scores of the participants were evaluated to ensure complete recovery of their knee joints. All the 331 setting of the running test was just the same as the playing basketball. In addition, Lysholm score is a 332 condition-specific score for evaluating knee ligament injury and widely used in various knee diseases. 333 It is simple, non-traumatic and easy to be accepted. IKDC has relatively high reliability, effectiveness 334 and sensitivity for the assessment of ligament injuries, especially anterior cruciate ligament injuries 335 and defects. Thus, it is suitable for evaluating post-exercise injuries of knee joint. 
ACL: anterior cruciate ligament; MCL: medial collateral ligament; LCL: lateral collateral ligament; PI: proportional-integral; BMI: body

Acknowledgments

340 The authors would like to doctors in the Department of Orthopedics, Fujian Medical University Union Hospital, for their assistance in

341 scale testing and experimental data collection.

\section{Authors' contributions}

343 JX, TZ: Conceptualization, Investigation, Supervision, Methodology, Formal analysis, Funding acquisition, Resources, Writing - original

344 draft, Writing - review \& editing, Visualization, Software. JC: Investigation, Data curation. XH: Investigation, Formal analysis. PW:

345 Investigation. All authors read and approved the final manuscript.

\section{Funding}

347 This study was supported by National Key Technologies Research and Development Program of China (2019YFC0118203), National 348 Natural Science Foundation of China (81701087), Fujian Provincial Joint Funds for the Innovation of Science and Technology 349 (2017Y9025), Outstanding Youth Fund of Fujian Agriculture and Forestry University (XJQ201820).

\section{$350 \quad$ Availability of data and materials}

351 The datasets used and analyzed during the current study are available from the corresponding author on reasonable request.

\section{Ethics approval and consent to participate}

353 Not applicable.

\section{Consent for publication}

355 Not applicable.

\section{Competing interests}

357 The authors declare that they have no competing interests.

358 Author details

$359{ }^{1}$ Department of Neurology, Fujian Institute of Geriatrics, Fujian Medical University Union Hospital, 29 Xinquan Road, 350001 Fuzhou,

360 China. ${ }^{2}$ Fujian Key Laboratory of Molecular Neurology, Fujian Medical University, 29 Xinquan Road, 350001 Fuzhou, China. ${ }^{3}$ Key

361 Laboratory of Brain Aging and Neurodegenerative Diseases, Fujian Medical University, 29 Xinquan Road, 350001 Fuzhou, China.

$362{ }^{4}$ College of Mechanical and Electrical Engineering, Fujian Agriculture and Forestry University, 350002 Fuzhou, China.

\section{References}

364 1. Zago M, Esposito F, Bertozzi F, Tritto B, Rampichini S, Galvani C, Galli M, Sforza C. Kinematic effects of repeated turns while running. European Journal of Sport Science. 2019; 19(8):1072-1081.

2. Notarnicola A, Maccagnano G, Barletta F, Ascatigno L, Astuto L, Panella A, Tafuri S, Moretti B. Returning to sport after anterior cruciate ligament reconstruction in amateur sports men: a retrospective study. Muscles, ligaments and tendons journal. 2016; 6(4):486-491. 
3. Chan LLY, Wong AYL, Wang MH. Associations between sport participation and knee symptoms: a cross-sectional study involving 3053 undergraduate students. BMC Sports Science, Medicine and Rehabilitation. 2020; 12(1):20.

4. Drew MK, Finch CF. The Relationship Between Training Load and Injury, Illness and Soreness: A Systematic and Literature Review. Sports Medicine. 2016; 46(6):861-883.

5. Bercovitz T, Herman A, Solomonow-Avnon D, Wolf A, Kodesh E. Plantar pressure modifications in experienced runners following an exhaustive run. Sports biomechanics. 2020:1-11.

6. Dannenmaier J, Kaltenbach C, Kolle T, Krischak G. Application of functional data analysis to explore movements: walking, running and jumping - a systematic review. Gait \& Posture. 2020; 77:182-189.

7. Tognetti A, Lorussi F, Carbonaro N, De Rossi D. Wearable Goniometer and Accelerometer Sensory Fusion for Knee Joint Angle Measurement in Daily Life. Sensors. 2015; 15(11):28435-28455.

8. Konrath JM, Karatsidis A, Schepers HM, Bellusci G, De Zee M, Andersen MS. Estimation of the knee adduction moment and joint contact force during daily living activities using inertial motion capture. Sensors. 2019; 19(7):1681.

9. Hu S, Dai M, Dong T, Liu T. A Textile Sensor for Long Durations of Human Motion Capture. Sensors. 2019; 19(10):2369.

10. Jaysrichai T, Suputtitada A, Khovidhungij W. Mobile Sensor Application for Kinematic Detection of the Knees. Annals of Rehabilitation Medicine. 2015; 39(4):599-608.

11. Bessone V, Petrat J, Schwirtz A. Ground Reaction Forces and Kinematics of Ski Jump Landing Using Wearable Sensors. Sensors. 2019; 19(9):2011.

12. Woo SLC, Debski RE, Withrow JD, Janaushek MA. Biomechanics of Knee Ligaments. American Journal of Sports Medicine. 1999; 27(4):533-543.

13. Woo SLC, Abramowitch SD, Kilger R, Liang R. Biomechanics of knee ligaments: injury, healing, and repair. Journal of Biomechanics. 2006; 39(1):1-20.

14. Ward E, Bodiwala GG, Thomas P. The importance of lower limb injuries in car crashes when cost and disability are considered. Accident Analysis \& Prevention. 1992; 24(6):613-620.

15. Almaawi A, Awwad W, Bamugaddam A, Alasheikh M, Muaddi M, Almutair O, Alomar AZ. Prevalence of knee injuries among male college students in Riyadh, Kingdom of Saudi Arabia. Journal of Orthopaedic Surgery and Research. 2020; 15(1):126.

16. Chen Y, Li JX, Hong Y, Wang L. Plantar Stress-Related Injuries in Male Basketball Players: Variations on Plantar Loads during Different Maximum-Effort Maneuvers. BioMed Research International. 2018; 2018:4523849-4523849.

17. Tian F, Li N, Zheng Z, Huang Q, Zhu T, Li Q, Wang W, Tsai T, Wang S. The effects of marathon running on three-dimensional knee kinematics during walking and running in recreational runners. Gait \& Posture. 2020; 75:72-77.

18. Khan PF, Sengottuvel S, Patel R, Gireesan K, Baskaran R, Mani A. Design and Implementation of a Discrete-Time Proportional Integral (PI) Controller for the Temperature Control of a Heating Pad. SLAS technology. 2018; 23(6):614-623.

19. Randsborg PH, Tajet J, Negård H, Røtterud JH. Manipulation under Anesthesia for Stiffness of the Knee Joint after Total Knee Replacement. Arthroplasty today. 2020; 6(3):470-474.

20. Niethammer TR, Altmann D, Holzgruber M, Goller S, Fischer A, Müller PE. Third generation autologous chondrocyte implantation is a good treatment option for athletic persons. Knee surgery, sports traumatology, arthroscopy : official journal of the ESSKA. 2020.

21. Roger J, Bertani A, Vigouroux F, Mottier F, Gaillard R, Have L, Rongièras F. ACL reconstruction using a quadruple semitendinosus graft with cortical fixations gives suitable isokinetic and clinical outcomes after 2 years. Knee surgery, sports traumatology, arthroscopy : official journal of the ESSKA. 2020.

22. Ciloglu O, Karaali E, Gorgulu FF, Ekiz T. Ultrasonographic evaluation of the patellar tendon length and elasticity after open-wedge high tibial osteotomy: A comparison with radiological and clinical parameters. The Knee. 2020; 27(4):1128-1134. 


\section{Figures}

(a)

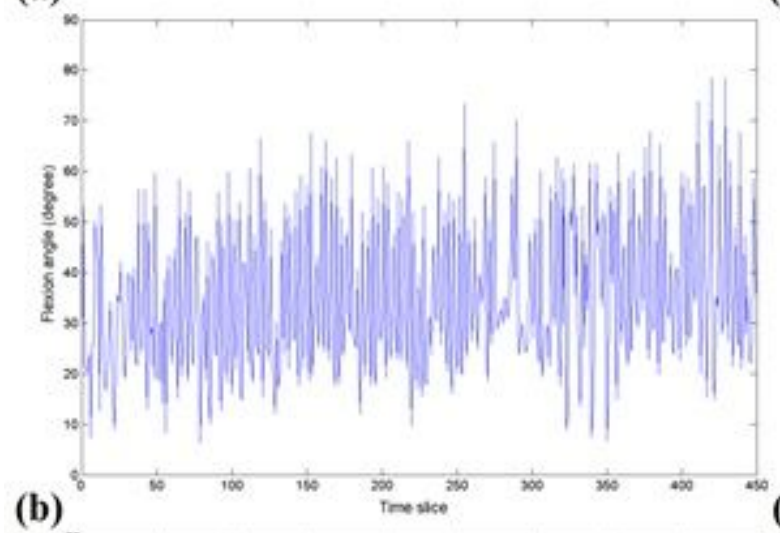

(b)

(c)

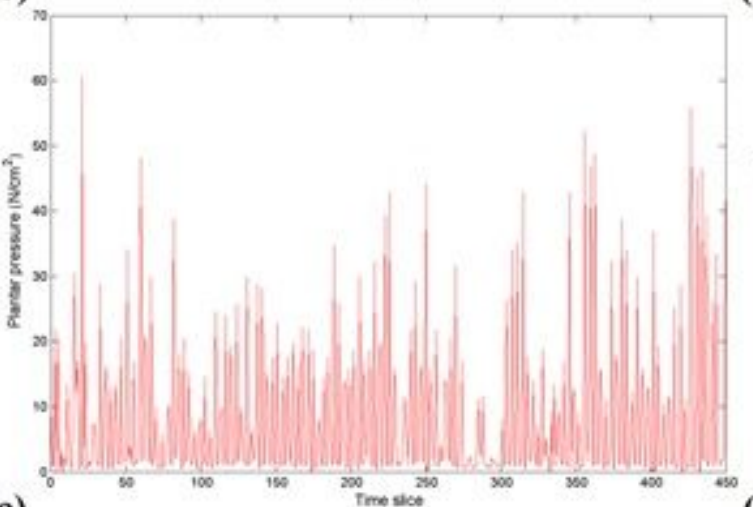

(d)
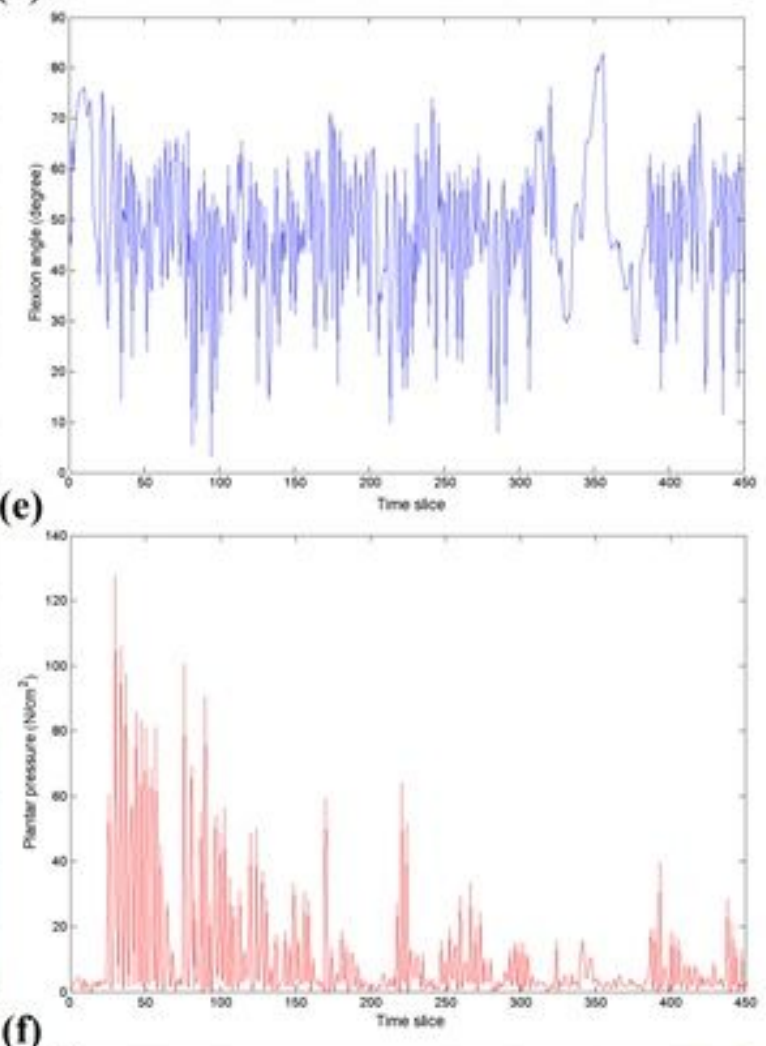

(f)

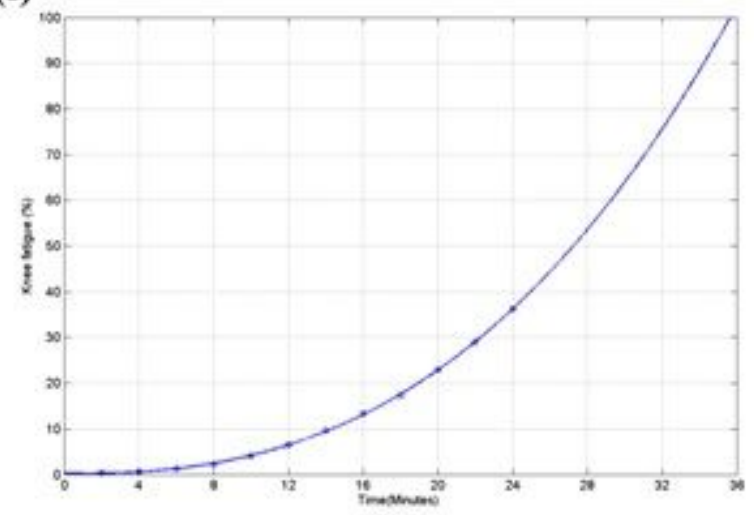

\section{Figure 1}

(a) Illustration of flexion angle data acquired by the device in running; (b) Illustration of plantar pressure data acquired by the device in running; (c) Illustration of predicting curve of knee fatigue in the 38th minute for running; (d) Illustration of flexion angle data acquired by the device in playing basketball; (e) Illustration of plantar pressure data acquired by the device in playing basketball; (f) Illustration of predicting curve of knee fatigue in the 24th minute for playing basketball. 


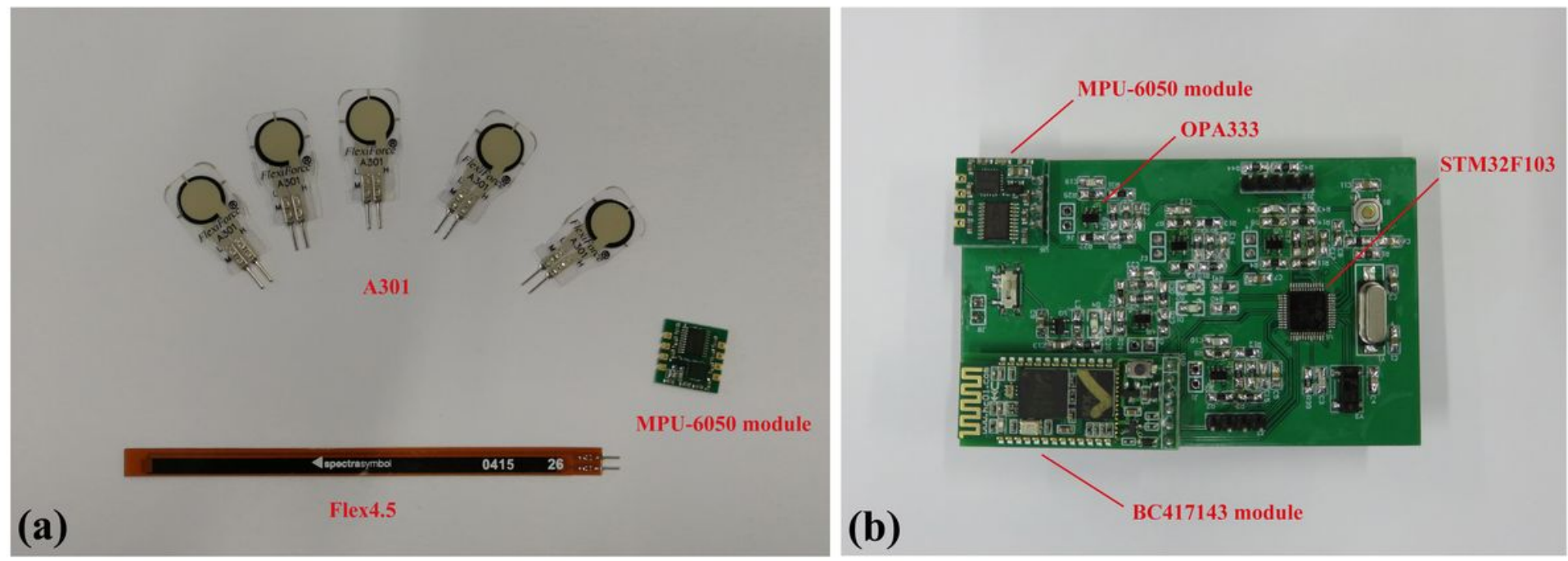

(c)
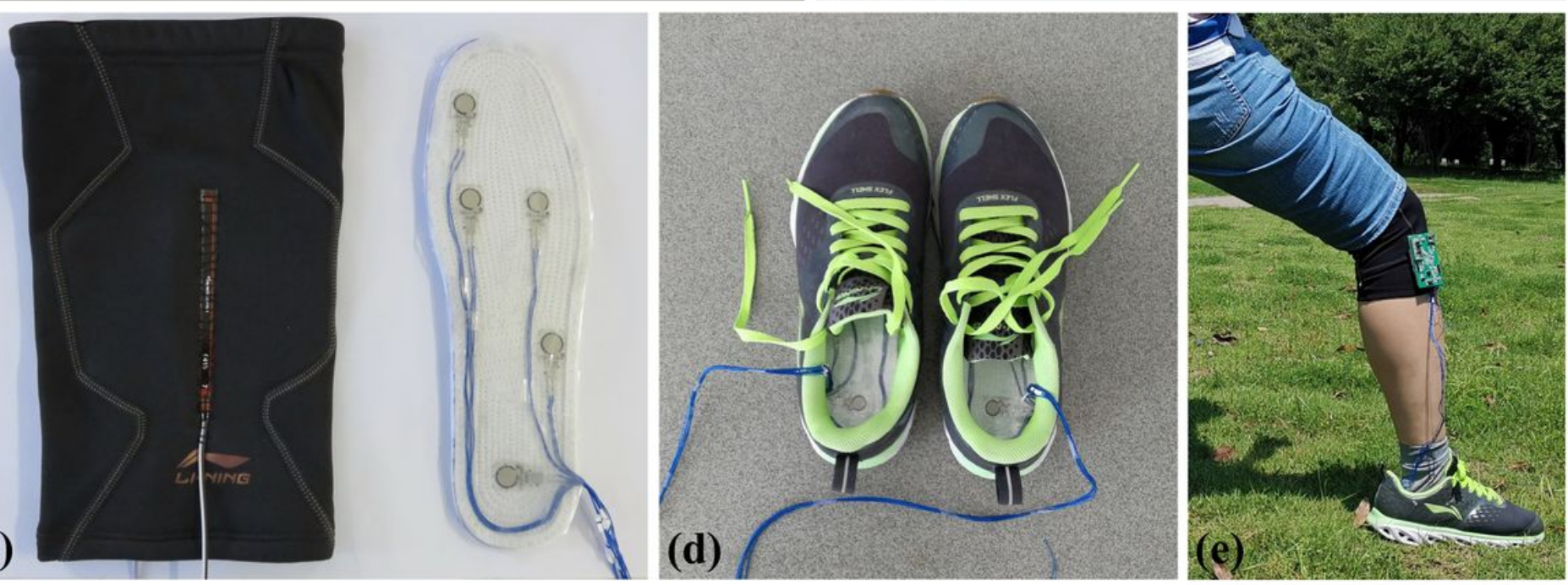

Figure 2

(a) The healthy time of different genders for knee joint during the four exercises without break; (b) The healthy time of different BMI situation for knee joint during the four exercises without break; (c) The healthy time for knee joint during the four exercises with and without break. $\left({ }^{*} p<0.05,{ }^{\star *} p<0.01\right.$, ${ }^{\star \star *} p<$ 0.001) 
(a)

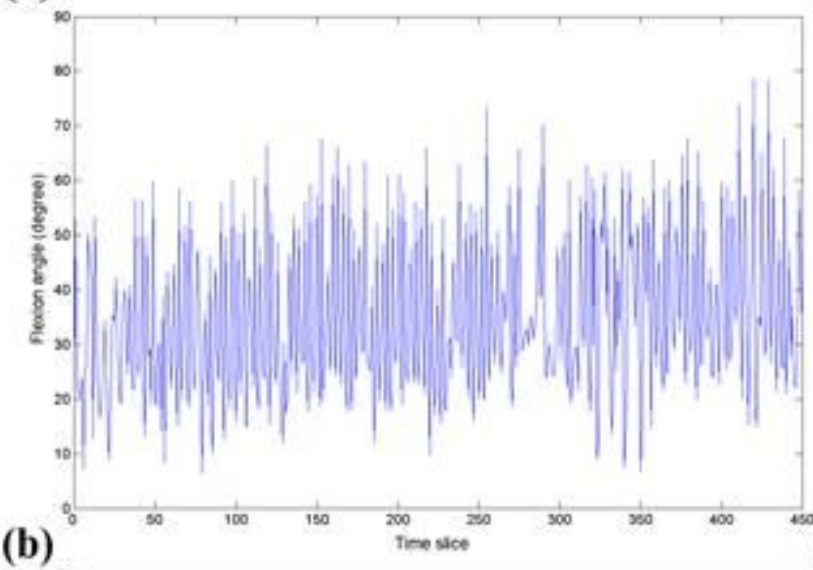

(b)

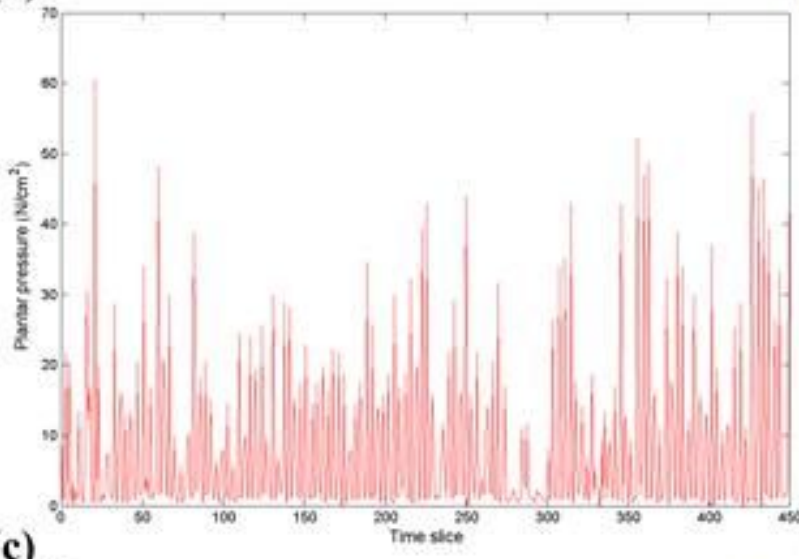

(c)

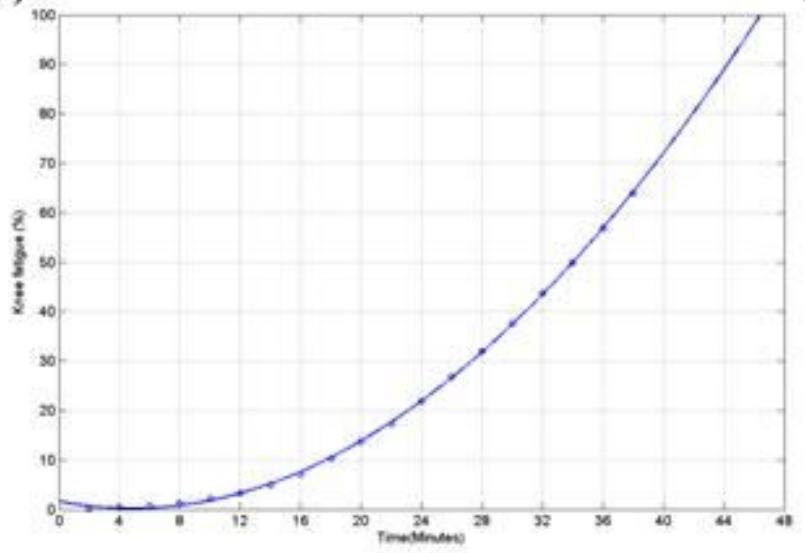

(d)

(e)
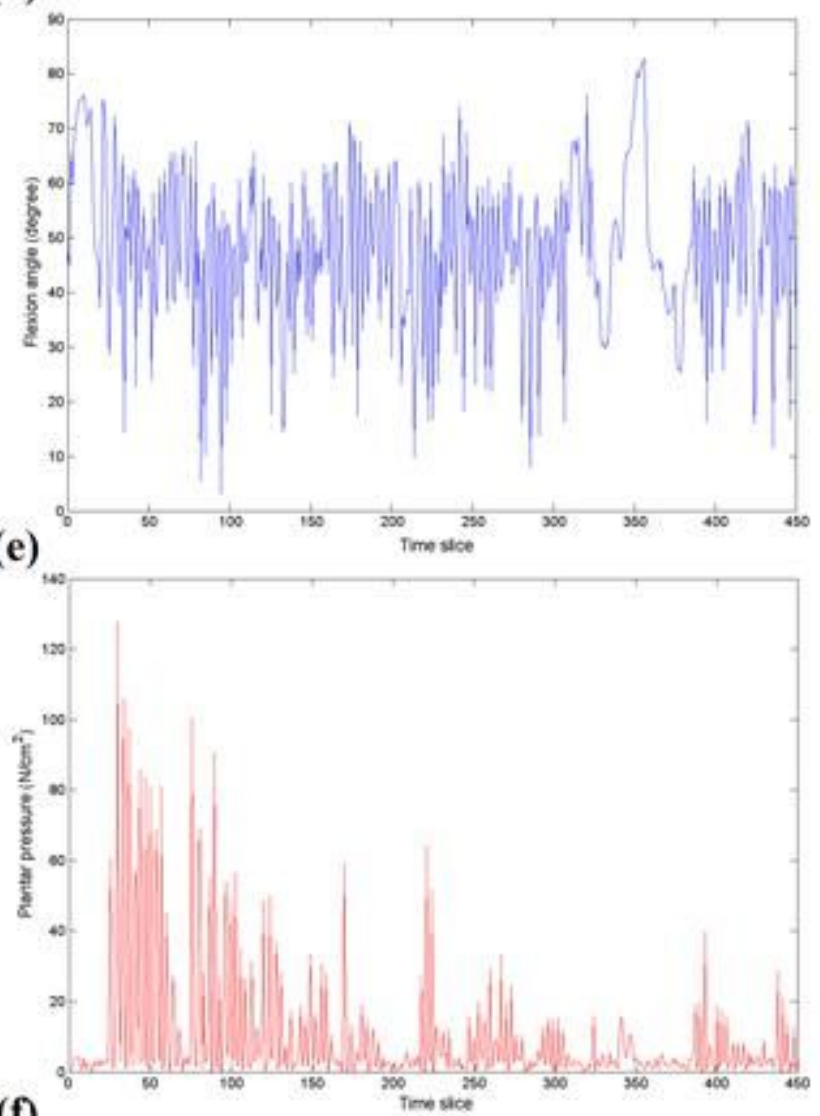

(f)

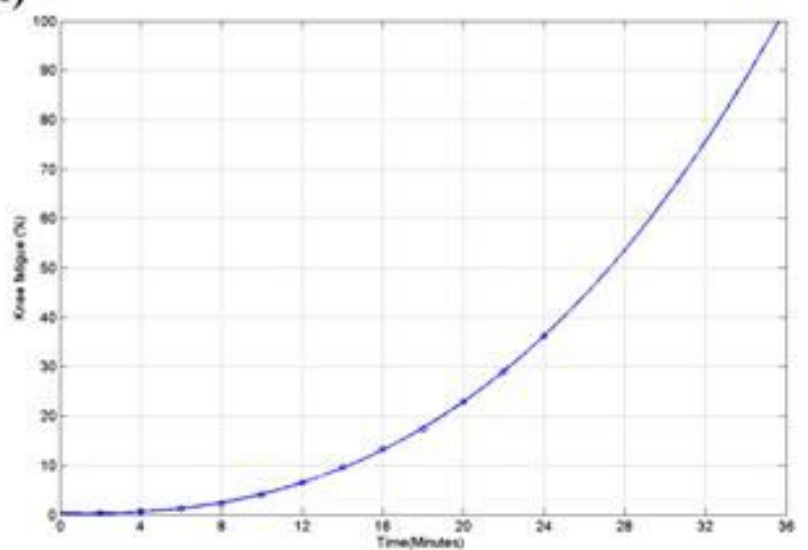

Figure 3

(a) Lysholm sores of testing and control groups before and after playing basketball and running; (b) IKDC sores of testing and control groups before and after playing basketball and running; (c) Lysholm sores of control group for different genders before and after playing basketball and running; (d) IKDC sores of control group for different genders before and after playing basketball and running; (e) Lysholm sores of control group for different BMI situation before and after playing basketball and running; (f) IKDC sores of control group for different BMI situation before and after playing basketball and running. $\left({ }^{*} p<0.05\right.$, ** $p<0.01, \star \star \star *<0.001)$ 
(a)

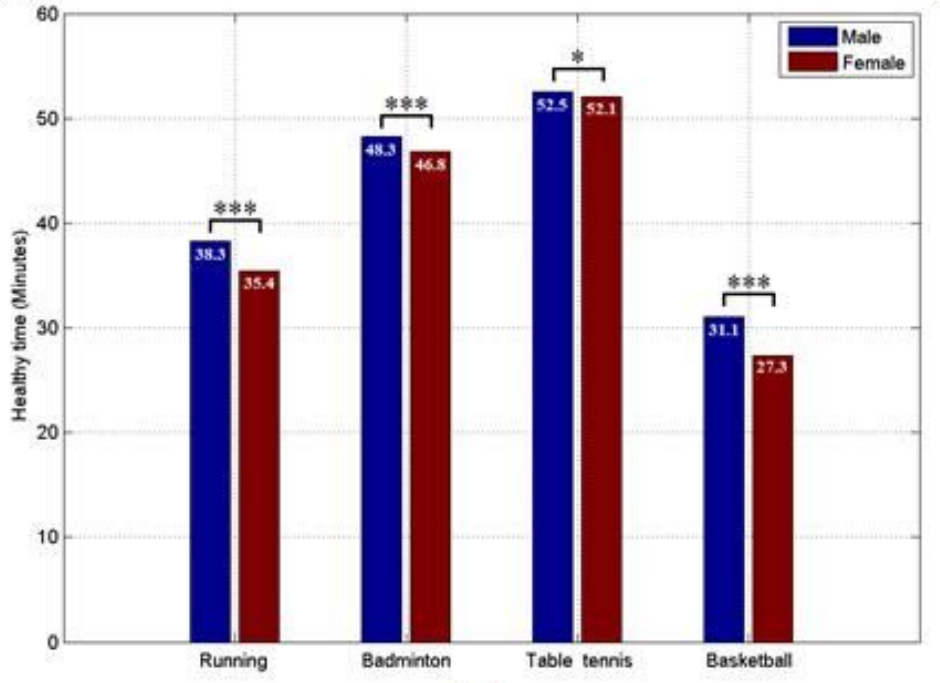

(b)

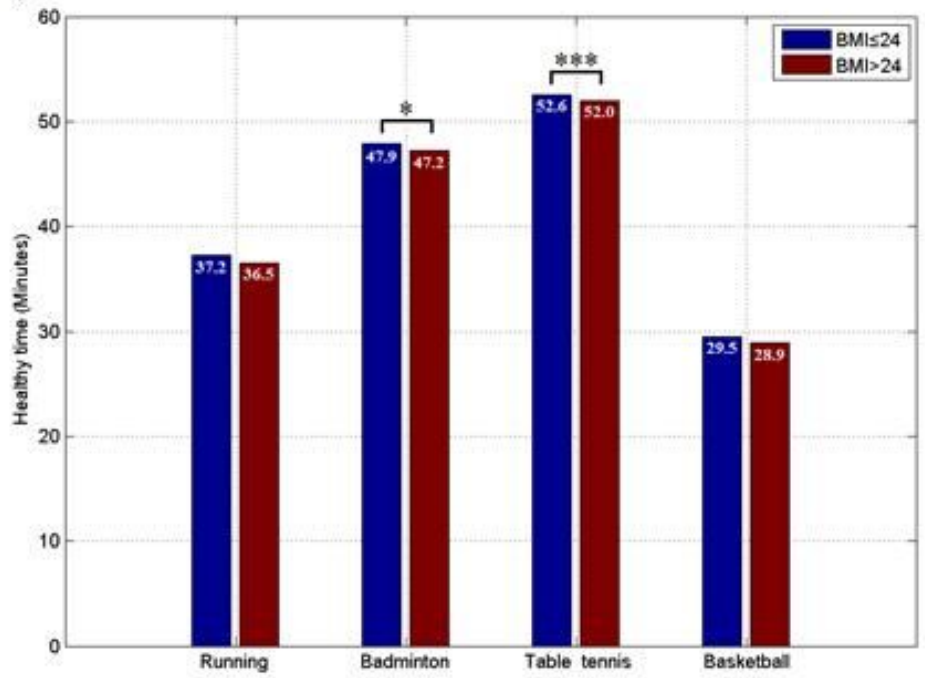

(c)

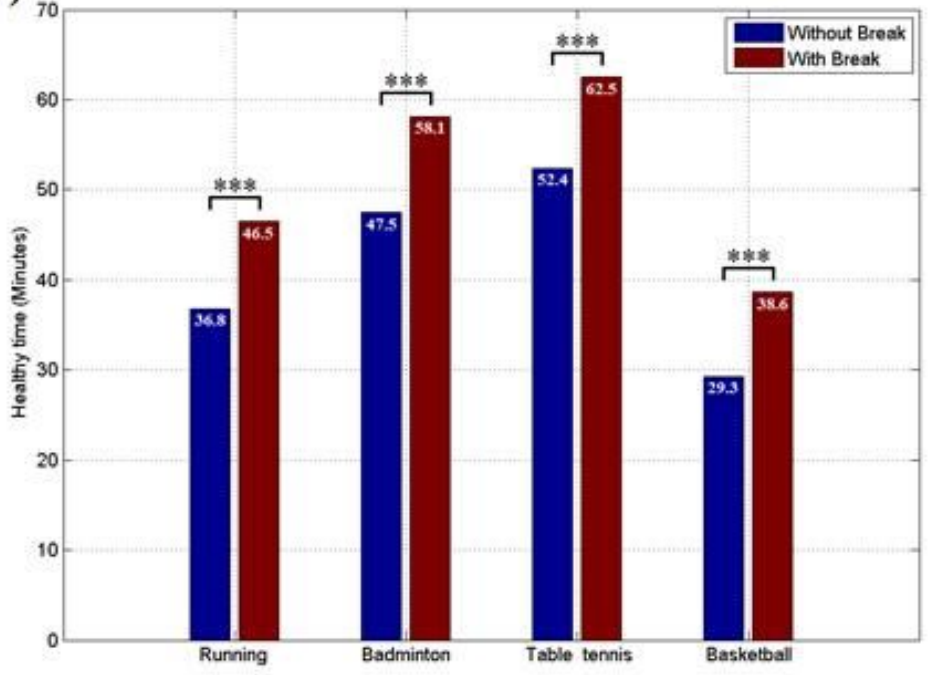

Figure 4

The sensing and predicting procedure for knee fatigue judgment. 
(a)

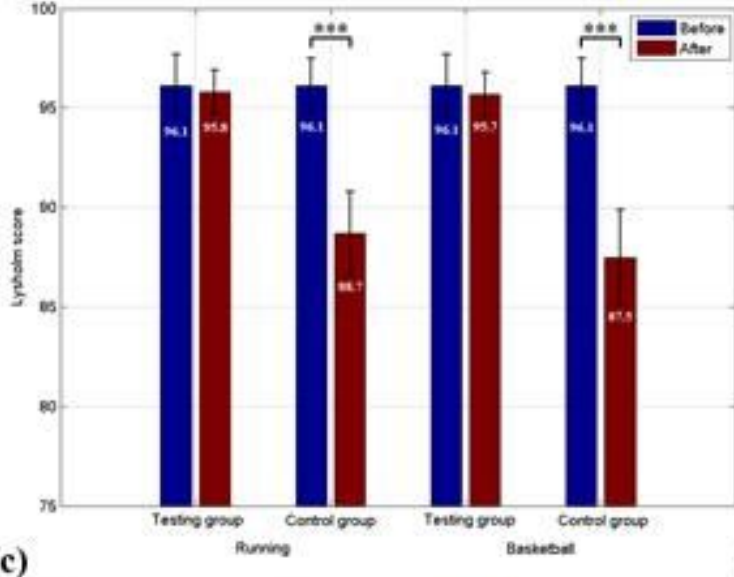

(c)

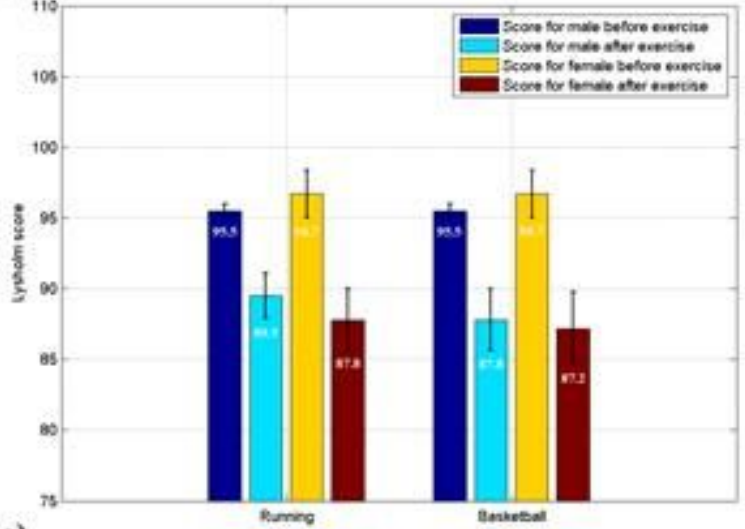

(e)

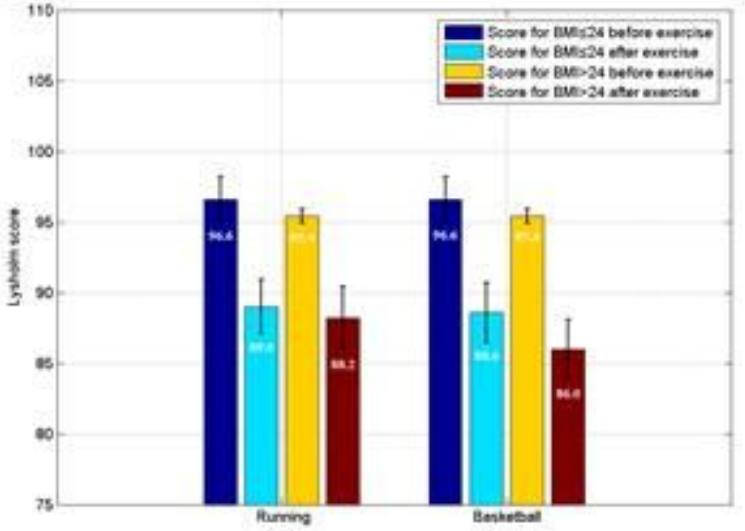

(b)

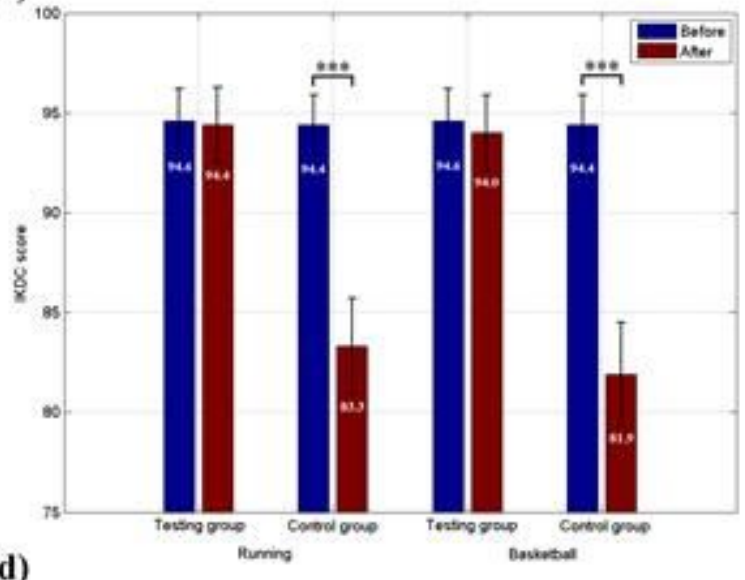

(d)

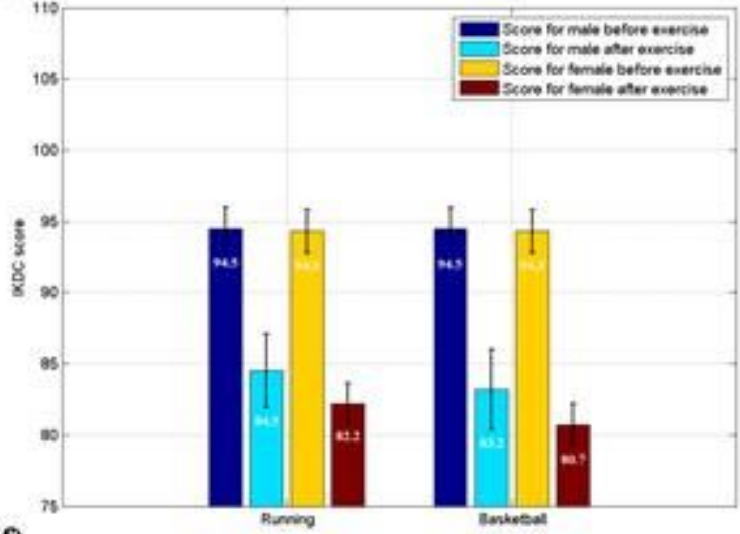

(f)

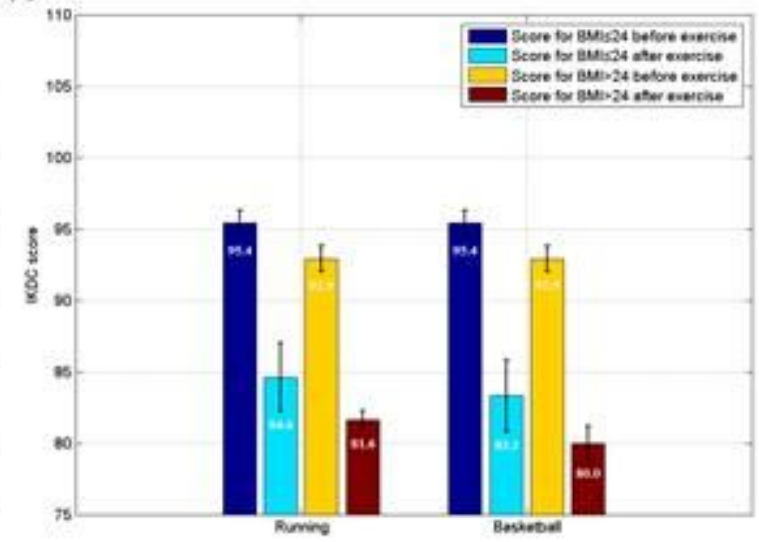

Figure 5

(a) Illustration of the three kinds of sensors used in the sensoring device; (b) Illustration of the hardware of the wearable device; (c) Illustration of the wearable accessories with sensors of the device; (d) Illustration of the shoes with sensors of the device; (e) Illustration of the wearing situation of the sensoring device. 